\title{
Urban Air Mobility_Challenges and Opportunities for Air Taxis
}

\author{
Carsten Rowedder ${ }^{(\varpi)}$ \\ Composite Technology Center GmbH, 21684 Stade, Germany \\ carsten.rowedderdairbus.com
}

Unfortunately, the translation of the script was not available for printing. The German original version is on page 49. 